\title{
Merosin-deficient congenital muscular dystrophy, autosomal recessive (MDC1A, MIM\#156225, LAMA2 gene coding for $\alpha 2$ chain of laminin)
}

\author{
Valérie Allamand ${ }^{* 1}$ and Pascale Guicheney ${ }^{1}$
}

\author{
${ }^{1}$ INSERM U523, Institut de Myologie-Bâtiment Joseph Babinski, Groupe Hospitalier Pitié-Salpétrière, 47 Boulevard \\ de l'Hôpital, 75651 Paris Cedex 13, France
}

Congenital muscular dystrophies (CMDs) are a highly heterogeneous group of neuromuscular disorders. A subgroup displays a specific deficiency in a protein of the extracellular matrix, the $\alpha \mathbf{2}$ chain of laminin-2 (merosin). A number of mutations in the gene encoding this protein have been identified in patients who present with a severe phenotype and white matter changes.

European Journal of Human Genetics (2002) 10, 91 -94. DOI: 10.1038/sj/ejhg/5200743

Keywords: congenital muscular dystrophy; laminin; merosin-deficiency; extracellular matrix; skeletal muscle

\begin{abstract}
Clinical definition
Congenital muscular dystrophies (CMD) are a group of neuromuscular disorders with severe muscle hypotonia at birth or within the first months of life, generalised muscle weakness, contractures of variable severity and delayed motor milestones. Histological changes in muscle biopsies consist of marked connective tissue proliferation, large variation in the size of the muscle fibres as well as some necrotic and regenerating fibres in early stages of the disease.

Merosin-deficient CMD is a severe form characterised by an absence of laminin $\alpha 2$ chain (formerly named merosin) around muscle fibres, elevated serum creatine kinase (CK), especially in the early months of life, no independent ambulation due to weakness and contractures, respiratory insufficiency which could need tracheotomy. Most patients have normal intelligence but some have been reported to show moderate mental retardation and epilepsy. They form a clinically homogeneous subgroup, in contrast to partial merosin deficiency cases who are less frequent, of variable severity but generally less severe. ${ }^{1}$
\end{abstract}

${ }^{*}$ Correspondence: V Allamand, INSERM U523, Institut de MyologieBâtiment Joseph Babinski, Groupe Hospitalier Pitié-Salpétrière, 47 Boulevard de l'Hôpital, 75651 Paris Cedex 13, France. Tel: 0142165720 Fax: 01421657 00; E-mail: v.allamand@myologie.chups.jussieu.fr Received 12 July 2001; accepted 31 August 2001

\section{Diagnosis}

It is usually made by the clinical features and muscle biopsy examination. Immunocytochemically, laminin $\alpha 2$ chain is deficient, with overexpression of $\alpha 4$ and $\alpha 5$ chains in the basal lamina surrounding myofibres. Integrin $\alpha 7 \beta 1$ and $\alpha$ dystroglycan, two functional laminin receptors show secondary reduction. ${ }^{2,3}$ The use of several antibodies directed against different regions of laminin $\alpha 2$ chain allows a precise distinction between complete and partial deficiency. ${ }^{4}$ Skin biopsies may be used instead of the muscle biopsy to confirm a complete laminin $\alpha 2$ chain deficiency.

Striking diffuse brain white matter changes in merosin deficient CMD patients generally appear after the first 6 to 12 months of life. Their detection by magnetic resonance imaging (MRI) has now become a very useful diagnostic tool, including in cases of partial deficiency.

Prenatal diagnosis can be made by determination of atrisk haplotypes using various intra- and extragenic markers, and also by immunocytochemical analysis of trophoblast, but only in the case of complete merosin deficiency. ${ }^{5}$

\section{Most important differential diagnoses}

Complete merosin deficiency is a well-defined entity without genetic heterogeneity, and its diagnosis is clear-cut. 
Partial merosin deficiency can be due to LAMA2 defects or to other causes. To our knowledge, all cases with partial merosin deficiency, normal intelligence and white matter changes are due to a primary LAMA2 defect. But a few cases with primary partial merosin deficiency may present a slight mental retardation, epilepsy, and/or very mild occipital cortex or cerebellum abnormalities in addition to the characteristic white matter changes. Their precise diagnosis is then difficult in absence of a molecular confirmation, as they can be mistaken for other disorders.

Some CMD cases with partial merosin deficiency and normal or subnormal intelligence without white matter changes are unlinked to LAMA2. They comprise cases with calf pseudohypertrophy and early respiratory failure for which a locus (MDC1B) has been assigned to chromosome $1 \mathrm{q} 42$, and also cases unlinked to $1 \mathrm{q} 42$ who do or do not develop severe macroglossia due to mutations in the Fukutinrelated protein gene. ${ }^{3,6}$

Partial deficiency observed also in Fukuyama CMD (mostly prevalent in Japan), Muscle-Eye-Brain disease, Walker-Warburg Syndrome is associated with moderate to severe mental retardation and brain and ocular abnormalities, which usually permit the diagnosis of these diseases.

\section{Disease frequency}

The prevalence of CMDs has been estimated to be $0.7 /$ 100000 in a sample from north-east Italy. ${ }^{7}$ Merosin-deficient CMD accounts for about $30 \%$ of the CMD cases in European countries, but only $6 \%$ in Japan.

\section{Gene}

The human LAMA2 gene is located on 6q22-23, spans over $260 \mathrm{~kb}$ and consists of 64 exons. mRNA is about $9.5 \mathrm{~kb}$.

\section{Function of the protein}

Laminins are a family of large extracellular trimeric glycoproteins. Five $\alpha$ chains, $3 \beta$ and $2 \gamma$ have been described to assemble and form at least 12 distinct heterotrimeric molecules. They form supramolecular structures by autoaggregating or by interacting with other components of the basal lamina. The C-terminal ends of all $\alpha$ chains contain domains crucial for their interaction with membrane receptors.

Laminin-2 and laminin-4 are composed of three chains: one heavy $(\alpha 2)$ and two light chains ( $\beta 1$ or $\beta 2$, respectively, and $\gamma 1$ ). They are expressed in the basal lamina of striated muscles and peripheral nerves. Laminin- 2 has been shown to be necessary for myogenesis as well as for the survival and stability of myotubes in vitro. A massive muscle fibre degeneration with scattered positive signals for apoptosis occurs in the very early stage of the disease. ${ }^{2}$
Laminin $\alpha 2$ chain is expressed in numerous tissues including skeletal muscle fibres, Schwann cells, synaptic basal lamina of peripheral nerves, heart, trophoblast and skin. The $\alpha 2$ chain is involved in cellular attachment, neurite growth and the migration of Schwann cells.

The $\alpha 2$ chain of laminin consists of six domains: I and II are part of the long arm; IIIa, IIIb and V contain cystein-rich EGFlike repeats and are predicted to have rigid rod-like structures; $\mathrm{IVa}, \mathrm{IVb}$ and VI are predicted to form globular structures. The globular C-terminal region $(\mathrm{G})$ is constituted of five internal homologous repeats and contains the interaction sites for membrane receptors that are, in striated muscle cells, $\alpha$ dystroglycan and integrin $\alpha 7 \beta 1{ }^{8}$

\section{Animal model}

The most commonly used animal models for merosindeficient CMD are the long-known spontaneous mouse strains $d y$ (dystrophia muscularis) and $d y^{2 \mathrm{~J}}$ which present a muscle pathology and a dysmyelination of the peripheral nervous system due to a complete and partial deficiency in the $\alpha 2$ chain of laminin, respectively. The $d y$ mouse is the most severely affected and the mutation responsible for its phenotype still remains unknown. The mouse $\alpha 2$ chain gene (Lama2) has been genetically linked to the same region of mouse chromosome 10 to which the $d y$ locus has been mapped. The mutation responsible for the $d y^{2 \mathrm{~J}}$ phenotype has been identified: it is a point mutation $(G \rightarrow A)$ in a consensus donor splice site leading to abnormal splicing and expression of six mRNA species. The M0 variant appears to be the predominant transcript and is translated into a protein lacking 57 in domain VI.

In addition, two knockout lines were generated $\left(d y^{3 \mathrm{~K}}\right.$ and $\left.d y^{\mathrm{W}}\right)$. These mice present a severe phenotype and have a reduced life span. ${ }^{9}$

\section{Mutations}

Since the identification of the first mutations leading to complete merosin deficiency in 1995, a number of mutations have subsequently been reported (Figure 1). ${ }^{2,5,10}$

Analysis of the laminin $\alpha 2$ chain cDNA or the LAMA2 gene itself showed that nucleotide substitutions, small deletions, or insertions induce complete merosin deficiency. Most of the mutations are localised in the $\mathrm{N}$-terminal domain (exons $1-31)$ and are predicted to produce truncated proteins. A 2 bp deletion, 2098delAC (703X), has been repeatedly found in several studies. ${ }^{2,5,10}$ A nonsense mutation, C967X, has also been identified in several Italian families. Some larger deletions have been reported.

The spectrum of the phenotypes of CMD patients with partial merosin deficiency is wide and caused by homozygous missense mutations, homozygous in-frame deletions, or a missense or an in-frame deletion associated to a nonsense mutation. Changes of conserved cystein residues 


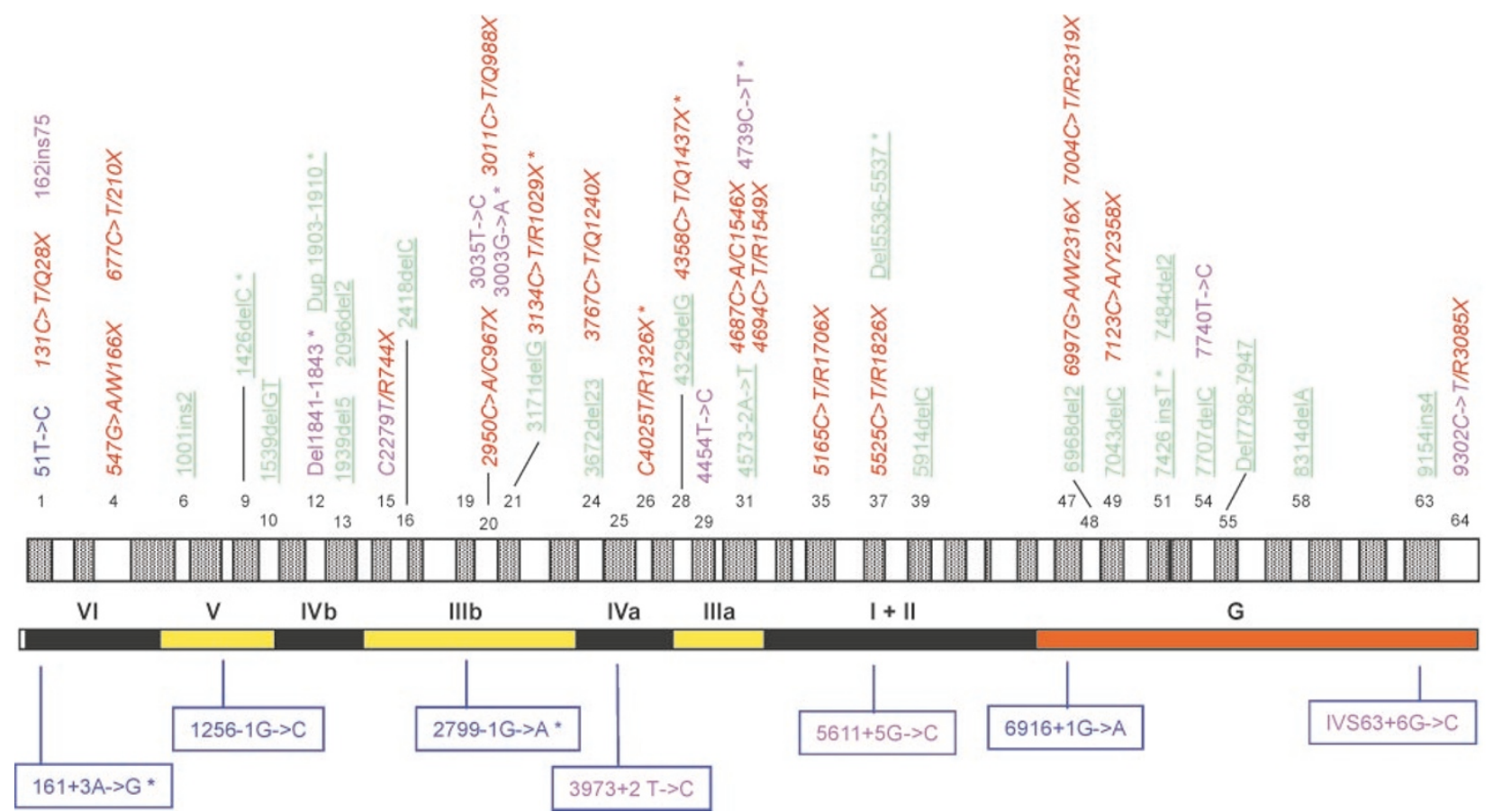

Figure 1 Schematic representation of all mutations in the LAMA2 gene causing merosin-deficient DMC. Mutations are scattered along the coding sequence containing 64 exons (only those containing mutations are numbered) and affect different domains of the protein which are represented below the coding sequence. Mutations are of different kinds: nonsense (in red, italicized), frameshift (in green, underlined) or splice site (in blue, boxed). Mutations causing a partial deficiency of the laminin $\alpha 2$ chain are indicated in purple. Asterisks refer to novel unpublished mutations identified by our group.

of the short arm of the protein induce partial deficiency probably by inducing proteolysis or instability of the scaffold.

Intronic and exonic primers have been designed to analyse LAMA 2 gene from cDNA or genomic $\mathrm{DNA}^{11}$ but the protein truncated test (PTT) is a more appropriate method to detect nonsense mutations in such a large gene. ${ }^{10}$

\section{Treatment}

To date no treatment available but the conditions of life are improved by physiotherapy to reduce contractures and arthrodesis to limit deformation. Ventilatory support and tracheotomy, when necessary, have contributed to a marked increase of the life expectancy for the most severely affected patients.

Two strategies have been tested in the murine models $d y$ and $d y^{2 \mathrm{~J}}$ to correct the phenotypes and have led to partial positive results. Myoblast transplantation, as well as transgenic experiments, have allowed renewed expression of the laminin $\alpha 2$ chain and amelioration of the muscle pathology to a certain extent but these techniques clearly cannot so far be applied to human. ${ }^{9}$

\section{References}

1 Tomé FMS: The saga of congenital muscular dystrophy. Neuropediatrics 1999; 30: 55-65.

2 Hayashi YK, Tezak Z, Momoi T et al: Massive muscle cell degeneration in the early stage of merosin-deficient congenital muscular dystrophy. Neuromuscul Disord 2001; 11: 350-359.

3 Muntoni F, Blake D, Brockington $\mathrm{M}$ et al: 85th ENMC International Workshop on Congenital Muscular Dystrophy 6th International CMD Workshop 1st Workshop of the MyoCluster Project 'GENRE' 27-28th October 2000, Naarden, The Netherlands. Neuromuscul Disord 2001; 12: 69-78.

4 He $\mathrm{Y}$, Jones $\mathrm{KJ}$, Vignier $\mathrm{N}$ et al: Mild congenital muscular dystrophy with primary partial laminin $\alpha 2$ chain deficiency: molecular study. Neurology 2001; 57: 1319-1322.

5 Guicheney P, Vignier N, Zhang X et al: PCR based mutation screening of the laminin alpha2 chain gene (LAMA2): application to prenatal diagnosis and search for founder effects in congenital muscular dystrophy. J Med Genet 1998; 35: 211 - 217.

6 Brockington M, Blake DJ, Prandini $\mathrm{P}$ et al: Mutations in the Fukutin-Related Protein Gene (FKRP) cause a form of congenital muscular dystrophy with secondary laminin $\alpha 2$ deficiency and abnormal glycosylation of $\alpha$-dystroglycan. Am J Hum Genet 2001; 69: 1198 - 1209 .

7 Mostacciuolo ML, Miorin M, Martinello F, Angelini C, Perini P, Trevisan CP: Genetic epidemiology of congenital muscular dystrophy in a sample from north-east Italy. Hum Genet 1996; 97: $277-279$. 
8 Miyagoe-Suzuki Y, Nakagawa M, Takeda S: Merosin and congenital muscular dystrophy. Microsc Res Tech 2000; 48: $181-191$.

9 Allamand V, Campbell KP: Animal models for muscular dystrophy : valuable tools for the development of therapies. Hum Mol Genet 2000; 9: 2459-2467.

10 Perogaro E, Marks H, Garcia CA et al: Laminin $\alpha 2$ muscular dystrophy. Genotype/phenotype studies of 22 patients. Neurology 1998; 51: $101-110$

11 Helbling-Leclerc A, Guicheney P: Analysis of the LAMA2 gene in merosin-deficient congenital dystrophy; in Anderson and Bushby, (eds). Methods of Molecular Medecine. Totowa: Humana Press Inc., 2000, pp. 199-218. 